\section{Glucocorticoid replacement in Addison disease}

\author{
Sirazum Choudhury and Karim Meeran
}

In a recent News \& Views by Lisa Muller and Marcus Quinkler (Imitating the cortisol profile improves the immune system. Nat. Rev. Endocrinol. 14, 137-139 (2018)) $)^{1}$, the authors suggest that optimum glucocorticoid replacement for the treatment of Addison disease is once-daily modified-release hydrocortisone (MR-hydrocortisone), instead of thrice-daily standard hydrocortisone. Standard hydrocortisone is administered thrice-daily because it has a short half-life. Prednisolone ${ }^{2}$, however, offers a cheaper once-daily replacement for the treatment of Addison disease, costing $£ 43$ per annum in the United Kingdom compared with $£ 2,900$ for MR-hydrocortisone. To date, randomized controlled trials comparing outcomes from different glucocorticoid replacement regimens in patients with adrenal failure have only been carried out with MR-hydrocortisone. The utility of cheaper drugs like prednisolone is unknown because of a reluctance to conduct expensive randomized controlled trials to test them.

For patients with Addison disease, the dose of glucocorticoid and the timing of treatment are crucial for achieving a satisfactory quality of life. If a patient does not receive a high enough dose of glucocorticoid at the right time, they are at increased risk of morbidity and mortality resulting from an Addisonian crisis. In the past, most clinicians have erred on the side of excess replacement to avoid such a crisis ${ }^{3-5}$ but the association of high doses of glucocorticoid with cardiovascular mortality is concerning.
In the present News \& Views article, Muller and Quinkler discuss a recent study by Andrea M. Isidori and colleagues ${ }^{6}$. The study by Isidori et al. reported that patients with adrenal insufficiency who were switched from thrice-daily hydrocortisone to once-daily MR-hydrocortisone had improved metabolic profiles and immune function compared with patients who continued on thrice-daily hydrocortisone.

Isidori and colleagues suggest that the administration of hydrocortisone late in the day (which is necessary on the thrice-daily routine) can be detrimental to health as it causes functional hypercortisolism every evening. MR-hydrocortisone has a lower bioavailability (and therefore a slower rate of absorption) than standard hydrocortisone, so it remains within the plasma for longer. It remains unclear whether improvements to metabolic profiles and immune function observed in the once-daily MR-hydrocortisone group resulted from a reduction of total steroid exposure or from reduced evening and nocturnal levels of steroid in the plasma, when peripheral steroid sensitivity is high. We would like to stress, however, that in this study, neither once-daily MR-hydrocortisone nor thrice-daily hydrocortisone was compared with once-daily prednisolone.

Prednisolone has been shown to have a plasma profile more similar to endogenous cortisol than any other steroid in healthy volunteers ${ }^{7}$. The drug is extremely cheap and widely available and it has a drug profile identical to that of MR-hydrocortisone. We now need a randomized controlled trial to compare once-daily prednisolone with oncedaily MR-hydrocortisone and thrice-daily hydrocortisone to determine which of the three has the best outcomes.

We believe that prednisolone, when used at the correct dose, might be the perfect replacement therapy in view of its very low cost, wide-spread availability and perfect plasma profile when taken once daily ${ }^{7}$. There is evidence that it is at least not inferior to the currently most widely used therapy, thrice-daily hydrocortisone ${ }^{2}$.

Sirazum Choudhury ${ }^{\prime}$ and Karim Meeran ${ }^{2 *}$ ${ }^{\prime}$ Metabolic Medicine, Charing Cross Hospital, London, UK.

${ }^{2}$ Imperial College, Endocrinology and Diabetes, Charing Cross Hospital, London, UK. *e-mail:k.meeran@imperial.ac.uk https://doi.org/10.1038/s41574-018-0049-6

1. Müller, L. \& Quinkler, M. Adrenal disease: imitating the cortisol profile improves the immune system. Nat. Rev. Endocrinol. 14, 137-139 (2018).

2. Smith, D., Prabhudev, H., Choudhury, S. \& Meeran, K. Prednisolone has the same cardiovascular risk profile as hydrocortisone in glucocorticoid replacement. Endocr. Connect. 6, 766-772 (2017).

3. Bergthorsdottir, R. et al. Visceral fat and novel biomarkers of cardiovascular disease in patients with Addison's disease: a case-control study. J. Clin. Endocrinol. Metab. 102, 4264-4272 (2017).

4. Mazziotti, G. et al. Management Of Endocrine Disease: Risk of overtreatment in patients with adrenal insufficiency: current and emerging aspects. Eur. J. Endocrinol. 177, R231-R248 (2017).

5. Hammarstrand, C. et al. Higher glucocorticoid replacement doses are associated with increased mortality in patients with pituitary adenoma. Eur. J. Endocrinol. 177, 251-256 (2017).

6. Isidori, A. M. et al. Effect of once-daily, modifiedrelease hydrocortisone versus standard glucocorticoid therapy on metabolism and innate immunity in patients with adrenal insufficiency (DREAM): a single-blind, randomised controlled trial. Lancet Diabetes Endocrinol. https://doi.org/10.1016/S22138587(17)30398-4 (2017)

7. Williams, E. L., Choudhury, S., Tan, T. \& Meeran, K. Prednisolone replacement therapy mimics the circadian rhythm more closely than other glucocorticoids. J. Appl. Lab. Med. 1, 152-161 (2016).

\section{Competing interests}

The authors declare no competing interests. 